\title{
On functions satisfying more than one equation of Schiffer type
}

\author{
by J. Macura and J. Śladkowska (Gliwice)
}

\begin{abstract}
The paper concerns properties of holomorphic functions satisfying more than one equation of Schiffer type ( $D_{n}$-equation). Such equations are satisfied, in particular, by functions that are extremal (in various classes of univalent functions) with respect to functionals depending on a finite number of coefficients.
\end{abstract}

Introduction. Let $S$ be the class of functions $f$ holomorphic and univalent in the unit disk $U$ with $f(0)=f^{\prime}(0)-1=0$, and let $V_{n}$ be the subset of $\mathbb{C}^{n-1}$ consisting of all points $\mathcal{A}_{n}=\left(a_{2}, \ldots, a_{n}\right)$ corresponding to the initial coefficients of some $f \in S$. It is known that for each $n \geq 2$ the coefficient region $V_{n}$ is simply connected and compact, and it coincides with the closure of its interior. Furthermore, to each $\mathcal{A}_{n} \in \partial V_{n}$ there corresponds a function $f \in S$ which satisfies a differential equation of the form

$$
\left(\frac{z w^{\prime}}{w}\right)^{2} P(w)=Q(z), \quad z \in U,
$$

where

$$
P(w)=\sum_{\nu=1}^{k-1} \frac{A_{\nu}}{w^{\nu}}, \quad Q(z)=\sum_{\nu=-k+1}^{k-1} \frac{B_{\nu}}{z^{\nu}},
$$

$A_{k-1} \neq 0, k \leq n, B_{0}>0, B_{-\nu}=\bar{B}_{\nu}, \nu=1, \ldots, k-1, Q(z) \geq 0$ everywhere on $\partial U$, and $Q(z)=0$ somewhere on $\partial U$. It is clear from (*) that $A_{k-1}=B_{k-1}$. An equation of the form $(*)$ where $P$ and $Q$ have all the indicated properties is called a $D_{n}$-equation of degree $k$. If $f$ is holomorphic near the origin and satisfies a $D_{n}$-equation of degree $k$, it must have the properties $f(0)=0,\left[f^{\prime}(0)\right]^{k-1}=1$. Any such function is called a $D_{n}$-function if it is holomorphic in $U$ and $f^{\prime}(0)=1$. It is known that every $D_{n}$-function is univalent $([6]$, p. 103) and there is a one-one correspondence between

1991 Mathematics Subject Classification: Primary 30C45.

Key words and phrases: univalent function, coefficient region, Schiffer type equation. 
$\partial V_{n}$ and the $D_{n}$-functions. Every $D_{n}$-function corresponds to some boundary point of $V_{n}$ and to any given boundary point of $V_{n}$ there corresponds a unique $D_{n}$-function. However, a $D_{n}$-function can satisfy more than one independent $D_{n}$-equation.

It seems natural to expect that points $\mathcal{A}_{n}$ for which the corresponding function $f$ satisfies more than one $D_{n}$-equation lie on an edge or vertex of the boundary surface while those points for which $f$ satisfies only one $D_{n^{-}}$ equation lie on a part of the surface which is in some sense more smooth. It is therefore of interest to investigate the class of functions corresponding to more than one $D_{n}$-equation. There are many surprisingly precise results about this class due to Schaeffer and Spencer [6], Kubota [2] and Bahtin [1].

Similar investigations can be carried out for other classes of univalent functions, e.g. for the class $S_{1}$ defined bellow.

Let $S_{1}$ consist of all functions of the form

$$
f(z)=b_{1} z+b_{2} z^{2}+\ldots, \quad z \in U,
$$

with $b_{1}>0$, univalent in $U$ and such that $f(U) \subset U$. The set of all points $\mathcal{B}_{n}=\left(b_{1}, \ldots, b_{n}\right)$ corresponding to functions of class $S_{1}$ forms a region $V_{n}$ in the $(2 n-1)$-dimensional space. Since $S_{1}$ becomes compact upon addition of the function $f(z)=0$, in the topology of uniform convergence on compact sets, the region $V_{n}$ is compact. It is known that to every $\mathcal{B}_{n}=\left(b_{1}, \ldots, b_{n}\right) \in$ $\partial V_{n}$ there corresponds an $f \in S_{1}$ which satisfies an equation of the form (*) with

$$
P(w)=\sum_{\nu=-k+1}^{k-1} \frac{A_{\nu}}{w^{\nu}}, \quad Q(z)=\sum_{\nu=-k+1}^{k-1} \frac{B_{\nu}}{z^{\nu}},
$$

where $A_{k-1} \neq 0, k \leq n, A_{-\nu}=\bar{A}_{\nu}, \nu=0, \ldots, k-1$, and $Q(z)$ has the same properties as in the case of $S$. It is also clear that $A_{k-1} / B_{k-1}=$ $b_{1}^{k-1}>0$. Also in this case, an equation of the form $(*)$, where $P(w)$ and $Q(z)$ have the properties indicated, is called a $D_{n}$-equation of degree $k$. If $f$ is holomorphic near the origin and satisfies a $D_{n}$-equation of degree $k$, it must have the properties $f(0)=0,\left[f^{\prime}(0)\right]^{k-1}=A_{k-1} / B_{k-1}$. A function $f$ which is holomorphic in $U$ and which has a positive derivative at the origin will be called a $D_{n}$-function if it satisfies a $D_{n}$-equation. It is known that every $D_{n}$-function belongs to $S_{1}$, and that there is a one-one correspondence between $\partial V_{n}$ without 0 and the $D_{n}$-functions [5].

Also in the case of the classes of Bieberbach-Eilenberg and GrunskySchah functions (the classes $B$ and $K$ ) it can be proved without difficulty that to each point of $\partial V_{n}$, where $V_{n}$ is the coefficient region constructed for $B$ or $K$, there corresponds a $D_{n}$-function, where the $D_{n}$-equation is of the form $(*)$ with $P(w)$ and $Q(z)$ of the form $(* *)$ with $A_{-\nu}=A_{\nu}$ for $B$ and 
$A_{-\nu}=(-1)^{\nu} \bar{A}_{\nu}$ for $K$, and $Q(z) \geq 0$ on $\partial U$. Just as for $S$, it is also here of interest to investigate the class of $D_{n}$-functions belonging to more than one $D_{n}$-equation.

The same problems for the classes $S_{1}$ and $B$ were investigated by one of the present authors in [7] and [4], by Jakubowski and Majchrzak in [3] and by Starkov in [8]. It turned out that not all the properties of the functions in $(*)$ were in fact used in the derivation of the majority of the results of [7] and [4]. The aim of this paper is to investigate functions satisfying two independent equations of the form $(*)$ under weaker assumptions on $P(w)$ and $Q(z)$. These assumptions are always satisfied for equations of the form (*) constructed for $S_{1}, B$ and $K$, and maybe for other classes of univalent functions.

I. Properties of functions satisfying more than one $D_{n}$-equation. Consider a differential equation of the form

$$
\left(\frac{z w^{\prime}}{w}\right)^{2} P(w)=Q(z)
$$

where

$$
\begin{gathered}
P(w)=\sum_{\substack{\nu=-k+1 \\
w^{\nu}}}^{k-1} \quad Q(z)=\sum_{\substack{\nu=-k+1 \\
z^{\nu}}}^{k-1} \frac{B_{\nu}}{k \geq 2, \quad z \in U=\{z:|z|<1\},}
\end{gathered}
$$

$\left|A_{-k+1}\right|=\left|A_{k-1}\right| \neq 0, B_{-\nu}=\bar{B}_{\nu}, \nu=1, \ldots, k-1, Q(z)$ is real and nonnegative on the circle $\partial U=\{z:|z|=1\}$ and $Q(z)=0$ somewhere on $\partial U$.

Let

$$
f(z)=b_{1} z+b_{2} z^{2}+\ldots, \quad 1 \neq b_{1}>0, z \in U,
$$

satisfy (1) in $U$. It follows from Theorem 4.1 of [9] that $f$ is bounded and univalent in $U$. Furthermore, it has an analytic continuation to $\partial U$ except for a finite number of points, where this continuation is only continuous. At these points

$$
f(z)=\sum_{k=m}^{\infty} c_{k}\left(z-z_{0}\right)^{k / n}, \quad n, m \in \mathbb{N} .
$$

The obvious necessary condition for the function (2) to satisfy (1) is that $A_{k-1}=b_{1}^{k-1} B_{k-1}$. The equation (1), by analogy to equations satisfied by extremal functions in various classes of univalent functions, will be called an equation of Schiffer type. In the case when $A_{-\nu}=\bar{A}_{\nu}, A_{-\nu}=A_{\nu}$ or $A_{-\nu}=(-1)^{\nu} \bar{A}_{\nu}, \nu=1, \ldots, k-1$, it can be proved ([5], [9], Th. 4.4) that 
$f$ is respectively a bounded function $(f(U) \subset U)$, a Bieberbach-Eilenberg function or a Grunsky-Schah function.

Suppose now that $f$, apart from (1), satisfies another equation of the same type,

$$
\left(\frac{z w^{\prime}}{w}\right)^{2} P_{1}(w)=Q_{1}(z)
$$

where

$$
P_{1}(w)=\sum_{\nu=-l+1}^{l-1} \frac{C_{\nu}}{w^{\nu}}, \quad Q_{1}(z)=\sum_{\nu=-l+1}^{l-1} \frac{D_{\nu}}{z^{\nu}}, \quad l \geq 2,
$$

$\left|C_{-l+1}\right|=\left|C_{l-1}\right| \neq 0, D_{-\nu}=\bar{D}_{\nu}, \nu=1, \ldots, l-1, Q_{1}(z)$ is real and nonnegative on $\partial U$. Suppose that $l>k$. The properties of $f$ are then given in Theorems 1-6.

THEOREM 1. The function $f$ can be continued to the entire plane as an algebraic function.

Proof. This follows by division of (1) by (3).

Let $F$ denote the algebraic function obtained in this way.

THEOREM 2. Both at 0 and at $\infty$, all analytic elements of $F$ only assume values 0 and $\infty$.

Proof. Suppose that an element of $F$ assumes a value $w_{0} \neq 0, \infty$ at 0 . Then this element has the form

$$
w=w(z)=w_{0}+\sum_{j=q}^{\infty} c_{j} z^{j / m},
$$

where $c_{q} \neq 0$, and $q \geq 1, m \geq 1$ are integers. Putting (4) into (1) or (3) and letting $z \rightarrow 0$, since $z w^{\prime}(z) / w(z) \rightarrow 0$, we obtain $0=\infty$ in both cases. In a similar way we obtain a contradiction when the element has centre $\infty$.

Theorem 3. All elements of $F$ with centre 0 and $\infty$ are smooth and invertible. Furthermore, the number of elements with centre 0 which assume value 0 at 0 and value $\infty$ at 0 , and the number of elements with centre $\infty$ which are 0 at $\infty$ and $\infty$ at $\infty$ do not exceed

$$
\min (k-1, l-k) \text {. }
$$

Proof. Let $w=w(z), w(0)=0$, be an element of $F$. Then

(6) $w^{l-k} \frac{A_{k-1}+\ldots+A_{-k+1} w^{2 k-2}}{C_{l-1}+\ldots+C_{-l+1} w^{2 l-2}}=z^{l-k} \frac{B_{k-1}+\ldots+\bar{B}_{k-1} z^{2 k-2}}{D_{l-1}+\ldots+\bar{D}_{l-1} z^{2 l-2}}$, 
where $A_{k-1}=b_{1}^{k-1} B_{k-1}$ and $C_{l-1}=b_{1}^{l-1} D_{l-1}$. Taking the $(l-k)$ th roots we obtain

$$
w\left(1+\lambda_{1} w+\ldots\right)=b_{1} \varepsilon_{j} z\left(1+\mu_{1} z+\ldots\right),
$$

where $\varepsilon_{j}=\exp \{i 2 \pi j /(l-k)\}, j=0, \ldots, l-k-1$. By the implicit function theorem, $w=w(z)$ is a smooth invertible element of the form

$$
w=b_{1} \varepsilon_{j}\left(z+c_{2}^{(j)} z^{2}+\ldots\right)
$$

and the number of different elements does not exceed $l-k$ (each determined by an $(l-k)$ th root of 1$)$. Likewise, an element with centre 0 such that $w(0)=\infty$ and

$$
\frac{1}{w^{l-k}} \frac{A_{-k+1}+\ldots+A_{k-1} w^{-2 k+2}}{C_{-l+1}+\ldots+C_{l-1} w^{-2 l+2}}=z^{l-k} \frac{B_{k-1}+\ldots+\bar{B}_{k-1} z^{2 k-2}}{D_{l-1}+\ldots+\bar{D}_{l-1} z^{2 l-2}},
$$

where $\left|A_{-k+1}\right|=\left|A_{k-1}\right|=b_{1}^{k-1}\left|B_{k-1}\right|$ and $\left|C_{-l+1}\right|=\left|C_{l-1}\right|=b_{1}^{l-1}\left|D_{l-1}\right|$, has the form

$$
w=b_{1}^{-1} \varepsilon_{j} \eta_{1}\left(z^{-1}+d_{0}^{(j)}+d_{1}^{(j)} z+\ldots\right),
$$

where $\varepsilon_{j}$ is as above and $\left|\eta_{1}\right|=1$. Considering elements with centre $\infty$, we conclude in a similar way that they have either the form

$$
w=b_{1}^{-1} \varepsilon_{j} \eta_{2}\left(z+e_{0}^{(j)}+e_{1}^{(j)} z^{-1}+\ldots\right), \quad\left|\eta_{2}\right|=1,
$$

or

$$
w=b_{1} \varepsilon_{j} \eta_{3}\left(z^{-1}+f_{2}^{(j)} z^{-2}+\ldots\right), \quad\left|\eta_{3}\right|=1,
$$

where each of the elements (8), (9) and (10) is determined by an $(l-k)$ th root of 1 . So the number of elements of each of these three forms does not exceed $l-k$. The same bound has been obtained in the case of elements of the form (7). On the other hand, each of the elements (7)-(10) satisfies both (1) and (3). In particular, it follows that $\varepsilon_{j}$ in (7)-(10) must be both a $(k-1)$ th and an $(l-1)$ th root of 1 . Hence we obtain $(5)$.

Corollary 1. The number of elements of $F$ with centre 0 is equal to the rang of multivalency of this function.

Remark 1. If $q$ denotes the greatest common divisor of $k-1$ and $l-1$, then the number of elements of each of the forms (7)-(10) does not exceed $q$.

COROLLARY 2. The algebraic function $F$ can be at most $2 q$-valued, where $q$ is the greatest common divisor of $k-1$ and $l-1$.

THEOREM 4. If $p$ denotes the number of elements of $F$ with centre 0 then $F$ satisfies an algebraic equation of the form

$$
P(z, w)=b_{p}(z) w^{p}+b_{p-1}(z) w^{p-1}+\ldots+b_{0}(z)=0,
$$


$b_{p}(z) \not \equiv 0$, where $b_{p}(z), \ldots, b_{0}(z)$ are polynomials in $z$ of degree at most $p$, at least one of them having degree $p$. These polynomials have no common factor of positive degree and $P(z, w)$ is irreducible as a polynomial in $w$, i.e. it cannot be represented as a product of two polynomials in $w$ of positive degrees whose coefficients are polynomials in $z$.

Proof. $F$ is an algebraic $p$-valued function, because all its elements with centre 0 are smooth, and hence it satisfies an equation of the form (11), where $P(z, w)$ is irreducible as a polynomial in $w$ and $b_{j}(z)$ have no common factor of positive degree. It remains to prove that the $b_{j}(z)$ are polynomials of degree at most $p$ and at least one of them has degree $p$. Let $F^{-1}$ denote the inverse function to $F$. All elements with centre 0 and $\infty$ are smooth and invertible, so their inverses are smooth elements of $F^{-1}$ with centres 0 and $\infty$. We shall prove that these are the only elements of $F^{-1}$ with centres 0 and $\infty$. Indeed, $F^{-1}$ is also an algebraic function and the element $z=f^{-1}(w)$ satisfying two equations of the form

$$
\left(\frac{w z^{\prime}}{z}\right)^{2} \sum_{j=-k+1}^{k-1} \frac{B_{j}}{z^{j}}=\sum_{j=-k+1}^{k-1} \frac{A_{j}}{w^{j}}
$$

and

$$
\left(\frac{w z^{\prime}}{z}\right)^{2} \sum_{j=-l+1}^{l-1} \frac{D_{j}}{z^{j}}=\sum_{j=-l+1}^{l-1} \frac{C_{j}}{w^{j}}
$$

belongs to this function. As in the case of $F$, it can be proved that elements with centre 0 can only assume values 0 and $\infty$ at 0 and, analogously, elements with centre $\infty$ can only assume values 0 and $\infty$ at $\infty$, and apart from this, they are invertible, so their inverses are elements with centres 0 and $\infty$ of the function $F$ (and these are all such elements). The number of the latter elements is $2 p$, so the number of elements with centres 0 and $\infty$ of $F^{-1}$ is $2 p$. Hence we conclude that there are $p$ elements with centre 0 and $p$ elements with centre $\infty$. Thus $F^{-1}$ is a $p$-valued function. Therefore $P(z, w)$ must be a polynomial of degree $p$ with respect to $z$, and this gives the assertion.

COROLlary 3. The number of elements of $F$ with centre 0 assuming value 0 at 0 is equal to the number of elements with centre $\infty$ assuming value $\infty$ at $\infty$. Hence the number of elements with centre 0 assuming value $\infty$ at 0 equals the number of elements with centre $\infty$ assuming value 0 at $\infty$.

Proof. Let $(a \rightarrow b)$ denote an element with centre $a$ assuming value $b$ at $a$. Let $\mu$ be the number of elements $(0 \rightarrow 0)$ and $\mu^{\prime}$ the number of elements $(\infty \rightarrow \infty)$, and suppose $\mu^{\prime}>\mu$. The number of elements $(0 \rightarrow \infty)$ is $p-\mu$, the number of elements $(\infty \rightarrow 0)$ is $p-\mu^{\prime}$. Therefore the number 
of elements of $F^{-1}$ with centre 0 is $\mu+p-\mu^{\prime}<\mu+p-\mu=p$, which is not the case. The proof in the case $\mu^{\prime}<\mu$ is analogous.

Theorem 5. The function $F$ is not odd-valued unless it is single-valued.

Proof. It is sufficient to prove that the number of elements with centre 0 is even. So it is sufficient to prove that the number of elements assuming value 0 at 0 is the same as the number of elements assuming value $\infty$ at 0 . Let the two numbers be $\mu$ and $\nu$ respectively. Suppose first that $\nu>0$ (we always have $\mu \geq 1$ ). The elements of the first group have the form

$$
w=b_{1} \varepsilon z+O\left(z^{2}\right),
$$

and those of the second are

$$
w=b_{1}^{-1} \varepsilon z^{-1}+O(1),
$$

where $|\varepsilon|=1$. Each of them satisfies an equation of the form

$$
P(z, w)=b_{p}(z) w^{p}+\ldots+b_{0}(z)=0, \quad \mu+\nu=p,
$$

where $b_{j}(z)=a_{0}^{(j)}+\ldots+a_{p}^{(j)} z^{p}$. From the Viète formulas we see that

$$
\frac{b_{\nu}(z)}{b_{p}(z)}=b_{1}^{-\nu} \eta z^{-\nu}+O\left(z^{-\nu+1}\right), \quad|\eta|=1,
$$

in the neighbourhood of 0 , that is,

$$
a_{0}^{(\nu)} z^{\nu}+\ldots+a_{p}^{(\nu)} z^{p+\nu}=\left(b_{1}^{-\nu} \eta+O(z)\right)\left(a_{0}^{(p)}+\ldots+a_{p}^{(p)} z^{p}\right) .
$$

Hence

$$
a_{0}^{(p)}=\ldots=a_{\nu-1}^{(p)}=0 .
$$

Consider now the elements with centre $\infty$. The number of elements with value $\infty$ at $\infty$ must be $\mu$, and the number of those with value 0 at $\infty$ must be $\nu$. These elements have the form

$$
w=b_{1}^{-1} \varepsilon z+O(1), \quad|\varepsilon|=1,
$$

and

$$
w=b_{1} \varepsilon z^{-1}+O\left(z^{-2}\right), \quad|\varepsilon|=1 .
$$

Using again the Viète formulas we obtain

$$
\frac{b_{\mu}(z)}{b_{p}(z)}=b_{1}^{-\mu} \eta z^{\mu}+O\left(z^{\mu-1}\right), \quad|\eta|=1,
$$

in the neighbourhood of $\infty$, that is,

$$
a_{0}^{(\mu)} z^{-\mu}+\ldots+a_{p}^{(\mu)} z^{p-\mu}=\left(a_{\nu}^{(p)} z^{\nu}+\ldots+a_{p}^{(p)} z^{p}\right)\left(b_{1}^{-\nu} \eta+O\left(z^{-1}\right)\right) .
$$

Hence

$$
a_{p}^{(p)}=a_{p-1}^{(p)}=\ldots=a_{\nu+1}^{(p)}=0 .
$$


From (12) and (13) we conclude that

$$
b_{p}(z)=a_{\nu}^{(p)} z^{\nu}
$$

Using once more the Viète formulas we have

$$
\frac{b_{0}(z)}{b_{p}(z)}=b_{1}^{\mu-\nu} \varepsilon z^{\mu-\nu}+O\left(z^{\mu-\nu+1}\right), \quad|\varepsilon|=1,
$$

in the neighbourhood of 0 , that is,

$$
a_{0}^{(0)}+\ldots+a_{p}^{(0)} z^{p}=a_{\nu}^{(p)} b_{1}^{\mu-\nu} \varepsilon z^{\mu}+O\left(z^{\mu+1}\right) .
$$

Similarly,

$$
\frac{b_{0}(z)}{b_{p}(z)}=b_{1}^{\nu-\mu} \eta z^{\mu-\nu}+O\left(z^{\mu-\nu-1}\right)
$$

in the neighbourhood of $\infty$, giving

$$
a_{0}^{(0)}+\ldots+a_{p}^{(0)} z^{p}=a_{\nu}^{(p)} b_{1}^{\nu-\mu} \eta z^{\mu}+O\left(z^{\mu-1}\right) .
$$

From (16) and (18) it follows that $a_{0}^{(0)}=\ldots=a_{\mu-1}^{(0)}=0$ and $a_{p}^{(0)}=\ldots=$ $a_{\mu+1}^{(0)}=0$, and hence

$$
b_{0}(z)=a_{\mu}^{(0)} z^{\mu}
$$

By (14), (19), (15) and (17),

$$
\frac{a_{\mu}^{(0)}}{a_{\nu}^{(p)}}=b_{1}^{\mu-\nu} \varepsilon=b_{1}^{\nu-\mu} \eta .
$$

Since $b_{1} \neq 1$, it follows that $\mu=\nu$.

Suppose now that $\nu=0$, i.e. the only elements with centre 0 are of the type $(0 \rightarrow 0)$, so they have the form $(\alpha)$. In this case $\mu=p \geq 1$. It follows from Corollary 3 that the only elements with centre $\infty$ are of the type $(\infty \rightarrow \infty)$, i.e. have the form $(\gamma)$. The number of these is, of course, also $p$. It follows from the Viète formulas that

$$
\frac{b_{0}(z)}{b_{p}(z)}=b_{1}^{p} \eta z^{p}+O\left(z^{p+1}\right), \quad|\eta|=1,
$$

in the neighbourhood of 0 , that is,

$$
a_{0}^{(0)}+\ldots+a_{p}^{(0)} z^{p}=z^{p}\left(b_{1}^{p} \eta+O(z)\right)\left(a_{0}^{(p)}+\ldots+a_{p}^{(p)} z^{p}\right) .
$$

Hence

$$
a_{0}^{(0)}=\ldots=a_{p-1}^{(0)}=0 \quad \text { or } \quad b_{0}(z)=a_{p}^{(0)} z^{p}, a_{p}^{(0)} \neq 0 .
$$

Using once more the Viète formulas, we have in the neighbourhood of $\infty$,

$$
\frac{b_{0}(z)}{b_{p}(z)}=b_{1}^{-p} \eta z^{p}+O\left(z^{p-1}\right), \quad|\eta|=1,
$$


that is,

$$
a_{0}^{(p)} z^{p}=z^{p}\left(b_{1}^{-p} \eta+O\left(z^{-1}\right)\right)\left(a_{0}^{(p)}+\ldots+a_{p}^{(p)} z^{p}\right)
$$

Hence

$$
a_{1}^{(p)}=\ldots=a_{p}^{(p)}=0 \quad \text { or } \quad b_{p}(z)=a_{0}^{(p)} \neq 0 .
$$

Let now $0<l \leq p$. Using again the Viète formulas, we have in the neighbourhood of 0 ,

$$
\frac{b_{p-l}(z)}{a_{0}^{(p)}(z)}=c z^{l}+O\left(z^{l+1}\right),
$$

and hence $a_{0}^{(p-l)}=\ldots=a_{l-1}^{(p-l)}=0$, and in the neighbourhood of $\infty$,

$$
\frac{b_{p-l}(z)}{a_{0}^{(p)}(z)}=d z^{l}+O\left(z^{l-1}\right)
$$

and hence $a_{l+1}^{(p-l)}=\ldots=a_{p}^{(p-l)}=0$. We have thus proved that

$$
b_{p-l}(z)=a_{l}^{(p-l)} z^{l} \quad \text { for } l=0,1, \ldots, p,
$$

and the polynomial $P(z, w)$ has the form

$$
P(z, w)=a_{0}^{(p)} w^{p}+a_{1}^{(p-1)} z w^{p-1}+\ldots+a_{l}^{(p-l)} z^{l} w^{p-l}+\ldots+a_{p}^{(0)} z^{p},
$$

and, in the case $p>1$, it is reducible, which is a contradiction. If $p=1$ then $f$ can be continued as a single-valued function. So the theorem has been proved.

COROLlary 4. If $F$ is a single-valued function it has the form

$$
F(z)=b_{1} z \text {. }
$$

Proof. In this case the equation (11) takes the form

$$
b_{1}(z) w+b_{0}(z)=0,
$$

where $b_{1}(z)=a_{0}^{(1)}, b_{0}(z)=a_{1}^{(0)} z$. From the fact that $w=f(z)$ satisfies this equation, we have $-a_{1}^{(0)} / a_{0}^{(1)}=b_{1}$ and $w=F(z)=b_{1} z$.

Remark 2. It has been proved by the way that

$$
b_{p}(z)=a_{\mu}^{(p)} z^{\mu} \quad \text { and } \quad b_{0}(z)=a_{\mu}^{(0)} z^{\mu},
$$

where $\mu=p / 2, p$ is even, and furthermore, $\left|a_{\mu}^{(p)}\right|=\left|a_{\mu}^{(0)}\right|$.

THEOREM 6. If $F$ is double-valued then each of its elements $w=w(z)$ satisfies the equation

$$
e^{i \alpha} w+e^{-i \alpha} w^{-1}=b_{1}^{-1}\left(e^{i \varphi} z-\frac{b_{2}}{b_{1}} e^{-i \alpha}+e^{-i \alpha} z^{-1}\right)
$$

Pr o of. This follows from Remark 2 and from the fact that $w=f(z)$ is an element of $F$. 
Remark 3. The conditions $Q(z) \geq 0, Q_{1}(z) \geq 0$ on $\partial U$ were not used in the proofs of Theorems $1-6$.

II. The case when one of the equations is of degree 3 . We are now concerned with the case when the equation (1) is of degree 3 . So it has the form

$$
\begin{aligned}
\left(\frac{z w^{\prime}}{w}\right)^{2}\left(\frac{A_{2}}{w^{2}}+\frac{A_{1}}{w}+A_{0}+A_{-1} w+A_{-2} w^{2}\right) & \\
= & \frac{B_{2}}{z^{2}}+\frac{B_{1}}{z}+B_{0}+\bar{B}_{1} z+\bar{B}_{2} z^{2}
\end{aligned}
$$

where the right-hand side is nonnegative on $\partial U$.

We assume additionally that the right-hand side of (22) has at least one zero on $\partial U$.

The number $l$ in (3) must of course be greater than 3 .

THEOREM 7. If a function $f$ of the form (2) satisfies (22) and (3) then it can be continued as an algebraic single-valued or double-valued function $F$.

Proof. It follows from Corollary 1 and Theorem 5 that $F$ can only be single-valued, double-valued or four-valued. We now exclude this last possibility.

Suppose that $F$ is four-valued. Then, by Theorem 4 and Remark 2, each element $w=w(z)$ of $F$ satisfies an equation of the form

$$
b_{4}(z) w^{4}+\ldots+b_{0}(z)=0,
$$

where

$$
\begin{gathered}
b_{\nu}(z)=a_{4}^{(\nu)} z^{4}+\ldots+a_{0}^{(\nu)}, \quad \nu=0, \ldots, 4, \\
b_{4}(z)=a_{2}^{(4)} z^{2}, \quad b_{0}(z)=a_{2}^{(0)} z^{2}, \\
a_{2}^{(4)} \neq 0, \quad a_{2}^{(0)} \neq 0, \quad\left|a_{2}^{(4)}\right|=\left|a_{2}^{(0)}\right|,
\end{gathered}
$$

and $b_{1}(z), b_{2}(z), b_{3}(z)$ are polynomials of degree at most 4 , at least one of them having degree 4 . By (7)-(10) and since $\varepsilon_{0}=1$ and $\varepsilon_{1}=-1$, elements of $F$ with centre 0 have the form

$$
w= \pm b_{1}\left(z+O\left(z^{2}\right)\right),
$$

and

$$
w= \pm b_{1}^{-1} \eta_{1}\left(z^{-1}+O(1)\right), \quad\left|\eta_{1}\right|=1,
$$

and elements with centre $\infty$ have the form

$$
w= \pm b_{1}^{-1} \eta_{2}(z+O(1)), \quad\left|\eta_{2}\right|=1,
$$

and

$$
w= \pm b_{1} \eta_{3}\left(z^{-1}+O\left(z^{-2}\right)\right), \quad\left|\eta_{3}\right|=1
$$


From the Viète formulas we now obtain $b_{3}(z)=a_{2}^{(3)} z^{2}$ and $b_{1}(z)=a_{2}^{(1)} z^{2}$, and so (23) takes the form

$$
A w^{2}+B w+C w^{-1}+D w^{-2}=P z^{2}+Q z+R+S z^{-1}+T z^{-2},
$$

where $A \neq 0, D \neq 0, P \neq 0, T \neq 0,|A|=|D|$. This equation is satisfied by each element of $F$. Denoting the left-hand side of (24) by $M(w)$ and the right-hand side by $N(z)$ we obtain

$$
M(w)=N(z) .
$$

If $w=w(z)$ is an arbitrary element of $F$ then

$$
M(w(z))=N(z)
$$

in the circle of this element. Differentiating (26) with respect to $z$ we have

$$
M^{\prime}(w(z)) w^{\prime}(z)=N^{\prime}(z) .
$$

Relations (22) and (26) give

$$
\left(\frac{z N^{\prime}(z)}{w M^{\prime}(w)}\right)^{2}\left(\frac{A_{2}}{w^{2}}+\ldots+A_{-2} w^{2}\right)=\frac{B_{2}}{z^{2}}+\ldots+\bar{B}_{2} z^{2} .
$$

Furthermore,

$$
w M^{\prime}(w)=2 A w^{2}+B w-C w^{-1}-2 D w^{-2},
$$

and by (25),

$$
\begin{aligned}
\left(w M^{\prime}(w)\right)^{2}= & \left(4 N^{2}(z)+\frac{B^{2}}{A} N(z)-2 B C-16 A D\right) \\
& -\left(4 B N(z)+\frac{B^{3}}{A}+8 A C\right) w \\
& -\left(4 C N(z)+8 B D+\frac{B^{2} C}{A}\right) \frac{1}{w} \\
& +\left(C^{2}-\frac{B^{2} D}{A}\right) \frac{1}{w^{2}} .
\end{aligned}
$$

Analogously, from (25) we have

$$
A_{-2} w^{2}=\frac{A_{-2}}{A}\left(N(z)-B w-C \frac{1}{w}-D \frac{1}{w^{2}}\right) .
$$

Putting (29) and (30) into (28) we conclude that each element of $F$ satisfies

$$
\begin{aligned}
\left(z N^{\prime}(z)\right)^{2}\left[\left(A_{2}\right.\right. & \left.-\frac{A_{-2} D}{A}\right)+\left(A_{1}-\frac{A_{-2} C}{A}\right) w \\
& \left.+\left(A_{0}+\frac{A_{-2}}{A} N(z)\right) w^{2}+\left(A_{-1}-\frac{A_{-2} B}{A}\right) w^{3}\right]
\end{aligned}
$$




$$
\begin{aligned}
= & \frac{S(z)}{z^{2}}\left[\left(C^{2}-\frac{B^{2} D}{A}\right)-\left(4 C N(z)+8 B D+\frac{B^{2} C}{A}\right) w\right. \\
& +\left(4 N^{2}(z)+\frac{B^{2}}{A} N(z)-2 B C-16 A D\right) w^{2} \\
& \left.-\left(4 B N(z)+\frac{B^{3}}{A}+8 A C\right) w^{3}\right],
\end{aligned}
$$

with $S(z)=B_{2}+B_{1} z+B_{0} z^{2}+\bar{B}_{1} z^{3}+\bar{B}_{2} z^{4}$, which is an equation of degree at most 3 with respect to $w$. In order that it could be an equation of the four-valued function $F$, the coefficients of all powers must vanish identically. This leads to the identities

$$
\begin{gathered}
\left(z N^{\prime}(z)\right)^{2}\left(A_{2}-\frac{A_{-2} D}{A}\right)=\frac{S(z)}{z^{2}}\left(C^{2}-\frac{B^{2} D}{A}\right) \\
\text { (b) }\left(z N^{\prime}(z)\right)^{2}\left(A_{1}-\frac{A_{-2} C}{A}\right)=-\frac{S(z)}{z^{2}}\left(4 C N(z)+8 B D+\frac{B^{2} C}{A}\right), \\
\text { (c) }\left(z N^{\prime}(z)\right)^{2}\left(A_{0}+\frac{A_{-2}}{A} N(z)\right) \\
=\frac{S(z)}{z^{2}}\left(4 N^{2}(z)+\frac{B^{2}}{A} N(z)-2 B C-16 A D\right), \\
\text { (d) } \quad\left(z N^{\prime}(z)\right)^{2}\left(A_{-1}-\frac{A_{-2} B}{A}\right)=-\frac{S(z)}{z^{2}}\left(4 B N(z)+\frac{B^{3}}{A}+8 A C\right) .
\end{gathered}
$$

Dividing now (b) and (d) by (c) we obtain

$$
\frac{A_{1}-A_{-2} C A^{-1}}{A_{0}+A_{-2} A^{-1} N(z)}=-\frac{4 C N(z)+8 B D+B^{2} C A^{-1}}{4 N^{2}(z)+B^{2} A^{-1} N(z)-2 B C-16 A D}
$$

and

$$
\frac{A_{-1}-A_{-2} B A^{-1}}{A_{0}+A_{-2} A^{-1} N(z)}=-\frac{4 B N(z)+8 A C+B^{3} A^{-1}}{4 N^{2}(z)+B^{2} A^{-1} N(z)-2 B C-16 A D},
$$

and hence $A_{1}=A_{-1}=0$ and

$$
C A_{0}+2 A_{-2} B D A^{-1}=0, \quad B A_{0}+2 A_{-2} C=0 .
$$

Then we have

$$
B^{2} D=A C^{2} .
$$

Suppose first that $B \neq 0$. From (32) and (31) we obtain $C \neq 0$ and $A_{0}=-2 A_{-2} C B^{-1}$, and from (32) and (a),

$$
A_{2}=A_{-2} D A^{-1}=A_{-2} C^{2} B^{-2},
$$


where $|C|=|B|$. In this case (22) takes the form

$$
A_{-2}\left(w-\frac{e^{i \varphi}}{w}\right)^{2}\left(\frac{z w^{\prime}}{w}\right)^{2}=\frac{S(z)}{z^{2}},
$$

where the right-hand side is nonnegative on $\partial U$.

Let now $B=0$. It follows from (32) that also $C=0$, and

$$
M(w)=A w^{2}+\frac{D}{w^{2}} .
$$

Putting $A / D=e^{-2 i \varphi}$ and $L(z)=A^{-1} N(z)$ in (c) we obtain

$$
A^{2}\left(z L^{\prime}(z)\right)^{2}\left(A_{0}+A_{-2} L(z)\right)=\frac{S(z)}{z^{2}}\left(4 A^{2} L^{2}(z)-16 A^{2} e^{2 i \varphi}\right),
$$

or

$$
\frac{\left(z L^{\prime}(z)\right)^{2}\left(A_{0}+A_{-2} L(z)\right)}{4\left(L(z)-2 e^{i \varphi}\right)\left(L(z)+2 e^{i \varphi}\right)}=\frac{S(z)}{z^{2}},
$$

where the only poles of the right-hand side are 0 and $\infty$.

Let $L\left(z_{j}\right)=2 e^{i \varphi}, j=1,2,3,4$, and $L\left(\zeta_{j}\right)=-2 e^{i \varphi}, j=1,2,3,4$, $z_{j}, \zeta_{j} \neq 0$. At these points either

$$
A_{0}+A_{-2} L(z)=0 \quad \text { or } \quad L^{\prime}(z)=0 .
$$

If $A_{0}+A_{-2} L(z)=0$ for at least one of these points then $A_{0}= \pm 2 A_{-2} e^{i \varphi}$ and by (a), $A_{2}=A_{-2} D A^{-1}=A_{-2} e^{2 i \varphi}$, so also in this case (22) takes the form (33):

$$
A_{-2}\left(w \pm \frac{e^{i \varphi}}{w}\right)^{2}\left(\frac{z w^{\prime}}{w}\right)^{2}=\frac{S(z)}{z^{2}}
$$

In the opposite case each of these points satisfies $L^{\prime}(z)=0$, so all roots of $L(z)-2 e^{i \varphi}$ and of $L(z)+2 e^{i \varphi}$ are at least double (because the first derivative at these roots is 0$)$ :

$$
\begin{aligned}
L(z)-2 e^{i \varphi} & =\frac{\lambda\left(z-z_{1}\right)^{2}\left(z-z_{2}\right)^{2}}{z^{2}}, \\
L(z)+2 e^{i \varphi} & =\frac{\mu\left(z-\zeta_{1}\right)^{2}\left(z-\zeta_{2}\right)^{2}}{z^{2}} .
\end{aligned}
$$

Differentiating (35) we obtain

$$
\begin{aligned}
z L^{\prime}(z) & =\frac{2 \lambda}{z^{2}}\left(z-z_{1}\right)\left(z-z_{2}\right)\left(z^{2}-z_{1} z_{2}\right) \\
& =\frac{2 \mu}{z^{2}}\left(z-\zeta_{1}\right)\left(z-\zeta_{2}\right)\left(z^{2}-\zeta_{1} \zeta_{2}\right) .
\end{aligned}
$$


From (35) and (36) we obtain

$$
\begin{aligned}
z L^{\prime}(z) & =\frac{2 \lambda}{z^{2}}\left(z^{4}-z_{1}^{4}\right), \\
L(z) \pm 2 e^{i \varphi} & =\frac{\lambda}{z^{2}}\left(z^{2} \pm z_{1}^{2}\right)^{2} .
\end{aligned}
$$

After substituting (37) into (34) we have

$$
A_{0}+A_{-2} L(z)=\frac{S(z)}{z^{2}}
$$

But, by the assumption, $S(z)$ has at least one root on the circle $\partial U$, and it is at least double. Denote this root by $z_{0}: S\left(z_{0}\right)=0, S^{\prime}\left(z_{0}\right)=0$. From (38) we have

$$
A_{0}+A_{-2} L\left(z_{0}\right)=0 \text { and } A_{-2} L^{\prime}\left(z_{0}\right)=0 .
$$

Therefore $z_{0}$ must be one of the points $z_{1}, z_{2}, \zeta_{1}, \zeta_{2}$, hence $L\left(z_{0}\right)= \pm 2 e^{i \varphi}$ and thus $A_{0}= \pm 2 A_{-2} e^{i \varphi}$, and we again obtain (33).

Consider now the consequences of (33) for $w=f(z)$. Put $w=f(z)$ in (33), where $z=e^{i t}, t \in[0,2 \pi)$. Since the right-hand side of (33) is nonnegative on $\partial U$ we have

$$
e^{2 i \alpha}\left(f\left(e^{i t}\right)-\frac{e^{i \varphi}}{f\left(e^{i t}\right)}\right)^{2}\left(\frac{e^{i t} f^{\prime}\left(e^{i t}\right)}{f\left(e^{i t}\right)}\right)^{2} \geq 0,
$$

where $2 \alpha=\arg A_{-2}$. Hence taking square roots gives

$$
\operatorname{Re}\left\{e^{i \alpha} \frac{i e^{i t} f^{\prime}\left(e^{i t}\right)}{f\left(e^{i t}\right)}\left(f\left(e^{i t}\right)-\frac{e^{i \varphi}}{f\left(e^{i t}\right)}\right)\right\}=0,
$$

and integration shows that

$$
\operatorname{Re}\left\{e^{i \alpha}\left(w+\frac{e^{i \varphi}}{w}\right)\right\}=c, \quad c=\text { const. }, w=f\left(e^{i t}\right) .
$$

We now investigate the set of points for which (39) is satisfied. Putting $w=e^{i \varphi / 2} \omega$ in (39) gives

$$
\operatorname{Re}\left\{e^{i \beta}\left(\omega+\frac{1}{\omega}\right)\right\}=c
$$

where $\alpha+\varphi / 2=\beta$. Putting $\omega=u+i v$ in (40) we obtain (41) $\quad u \cos \beta\left(\left(u^{2}+v^{2}\right)^{-2}+1\right)-v \sin \beta\left(1-\left(u^{2}+v^{2}\right)^{-2}\right)=c$.

Let $f_{1}(z)=e^{-i \varphi / 2} f(z)$. Then $f_{1}$ is also univalent and bounded and $\partial f_{1}(U)$ is the rotation of $\partial f(U)$ through the angle $-\varphi / 2$. So $\partial f_{1}(U)$, and likewise $\partial f(U)$, must intersect both coordinate axes in at least two points, and there must exist at least two points of intersection of opposite signs on each axis. If $(u, 0) \in \partial f_{1}(U)$ then

$$
u \cos \beta\left(u^{-2}+1\right)=c .
$$


If $\cos \beta \neq 0$ then

so

$$
u^{-1}+u=\frac{c}{\cos \beta},
$$

$$
u^{2}-\frac{c}{\cos \beta} u+1=0 .
$$

This is impossible because this equation has no solutions of opposite signs. Therefore $\cos \beta=0$, and hence $\sin \beta= \pm 1$ and (41) takes the form

$$
v\left(1-\left(u^{2}+v^{2}\right)^{-2}\right)=0 .
$$

Then $\partial f_{1}(U)$ consists of the circle $\partial U$ and of one or two slits extending from \pm 1 and lying on the $u$ axis, and $\partial f(U)$ consists of $\partial U$ and of slits extending from $\pm e^{i \varphi / 2}$ and lying on the line through 0 .

We now prove that $f$ can be continued as a double-valued function. In fact, if $f(U)=U$ then $f(z)=z$, which is not true because $b_{1} \neq 1$. Suppose now that $f(U)=U-L$ where $L$ is a segment extending from one of the points $\pm e^{i \varphi / 2}$ and lying on the line through $0: L=\left\{w: w= \pm e^{i \varphi / 2}(1-t)+t d\right\}$, $0 \leq t \leq 1$. Let $f\left(e^{i t_{0}}\right)=d$; hence $f^{\prime}\left(e^{i t_{0}}\right)=0$ and by (33), $e^{i t_{0}}$ is an at least double root of $S(z)$. Thus $S(z)$ has the form

$$
S(z)=\bar{B}_{2}\left(z-e^{i t_{0}}\right)^{2}\left(z-z_{0}\right)\left(z-\bar{z}_{0}^{-1}\right),
$$

where $\left|z_{0}\right| \leq 1$. If $\left|z_{0}\right|<1$ then $f\left(z_{0}\right)= \pm e^{i \varphi / 2}$, which is impossible because no point of $U$ is mapped to $\partial f(U)$. So $\left|z_{0}\right|=1$. Let $z_{0}=e^{i t_{1}}$ and

$$
S(z)=\bar{B}_{2}\left(z-e^{i t_{0}}\right)^{2}\left(z-e^{i t_{1}}\right)^{2} .
$$

In the case when $\partial f(U)$ consists of $\partial U$ and two slits, an analogous reasoning leads to the same result. Putting now (42) into (33) and taking square roots we obtain

$$
\sqrt{A_{-2}}\left(\frac{e^{i \varphi}}{w^{2}}-1\right) w^{\prime}=\sqrt{\bar{B}_{-2}}\left(1-\left(e^{i t_{0}}+e^{i t_{1}}\right) \frac{1}{z}+e^{i\left(t_{0}+t_{1}\right)} \frac{1}{z^{2}}\right) .
$$

Integration yields

(43) $-\sqrt{A_{-2}}\left(\frac{e^{i \varphi}}{w}+w\right)=\sqrt{\bar{B}_{-2}}\left(z-\left(e^{i t_{0}}+e^{i t_{1}}\right) \log z-e^{i\left(t_{0}+t_{1}\right)} \frac{1}{z}\right)+c$.

Of course, $e^{i t_{0}}+e^{i t_{1}}=0$, and (43) is an equation of a double-valued function, which was to be proved.

\section{References}

[1] A. K. Bahtin, Some properties of functions of class S, Ukrain. Mat. Zh. 33 (1981), 154-159 (in Russian); English transl.: Ukrainian Math. J. 33 (1981), 122-126.

[2] Y. Kubota, On extremal problems which correspond to algebraic univalent functions, Kodai Math. Sem. Rep. 25 (1973), 412-428. 
[3] Z. J. Jakubowski and W. Majchrzak, On functions realizing the maximum of two functionals at a time, Serdica 10 (1984), 337-343.

[4] A. Rost and J. Śladkowska, Sur les fonctions de Bieberbach-Eilenberg satisfaisant à plus qu'une équation de type de Schiffer, Demonstratio Math., to appear.

[5] H. L. Royden, The coefficient problem for bounded schlicht functions, Proc. Nat. Acad. Sci. U.S.A. 35 (1949), 637-662.

[6] A. C. Schaeffer and D. C. Spencer, Coefficient Regions for Schlicht Functions, Amer. Math. Soc., 1950.

[7] J. Śladkowska, Sur les fonctions univalentes, bornées, satisfaisant deux au moins $D_{n}$-équations, Demonstratio Math. 11 (1978), 1-28.

[8] V. V.Starkov, Bounded univalent functions realizing the extrema of two coefficients functionals at a time, in: Proc. XIth Instructional Conf. on the Theory of Extremal Problems, Łódź 1990, 31-33.

[9] K. Tochowicz, Functions which satisfy the differential equation of Schiffer type, Zeszyty Nauk. Politechn. Rzeszowskiej Mat. Fiz. 38 (6) (1987), 103-113.

INSTITUTE OF MATHEMATICS

SILESIAN TECHNICAL UNIVERSITY

ZWYCIȨSTWA 42

44-100 GLIWICE, POLAND 